\title{
Multivariable Predictive Control with Filtered Variables in Prediction Equations
}

\author{
Marek Kubalcik $^{1, a}$ and Vladimir Bobal ${ }^{1}$ \\ ${ }^{1}$ Tomas Bata University in Zlin, Faculty of Applied Informatics, Nam. T. G. Masaryka 5555, 76005 Zlin, Czech Republic
}

\begin{abstract}
The paper is focused on an implementation of a multivariable predictive controller with a colouring filter $C$ in a disturbance model. The filter is often essential for practical applications of predictive control based on input-output models. It is commonly considered as a design parameter because it has direct effects on closed loop performance. In this paper a computation of predictions for the case with the colouring filter is introduced. The computation is based on a particular model of the controlled system in the form of matrix fraction which is commonly used for description of a range of multivariable processes. Performance of closed loop system with and without the colouring filter in the disturbance model was compared.
\end{abstract}

\section{Introduction}

Typical technological processes require the simultaneous control of several variables related to one system. Each input may influence all system outputs. The design of a controller for such a system must be quite sophisticated if the system is to be controlled adequately. Simple decentralized PI or PID controllers largely do not yield satisfactory results. There are many different advanced methods of controlling multi-input-multi-output (MIMO) systems. The problem of selecting an appropriate control technique often arises. Perhaps the most popular way of controlling MIMO processes is by designing decoupling compensators to suppress the interactions [1] and the designing multiple SISO controllers [2] . This requires determining how to pair the controlled and manipulated variables. One of the most effective approaches to control of multivariable systems is model predictive control (MPC) [3], [4], [5]. An advantage of model predictive control is that multivariable systems can be handled in a straightforward manner. When using most of other approaches, the control actions are taken based on past errors. MPC uses also future values of the reference signals. It is essentially based on discrete or sampled models of processes. Computation of appropriate control algorithms is then realized especially in the discrete domain. The basic idea of the generalized predictive control [6], [7] is to use a model of a controlled process to predict a number of future outputs of the process. A trajectory of future manipulated variables is given by solving an optimization problem incorporating a suitable cost function and constraints. Only the first element of the obtained control sequence is applied. The whole procedure is repeated in following sampling period. This principle is known as the receding horizon strategy.

\footnotetext{
a Corresponding author: kubalcik@fai.utb.cz
}

An implementation of a multivariable predictive controller based on a matrix fraction model with a colouring filter $C$ in a disturbance model is described in this paper. The filter is often essential for practical applications of predictive control based on input-output models. Surveys of practical applications of predictive control are presented in [8], [9], [10]. It is commonly considered as a design parameter because it has direct effects on closed loop performance. A computation of predictions for the case with the colouring filter is introduced. The computation is based on a particular model of the controlled system in the form of matrix fraction which is commonly used for description of a range of processes.The filtering of variables is the equivalent of the colouring filter in the noise model. It is practically very difficult to estimate the coefficients of the colouring filter. A model with the $C$-filter is then utilized as an example with filtering of input and output variables when the filter $C$ is a tuning parameter. In the paper are derived prediction equations for an input-output model in the form of matrix fraction both for the case with the $C$-filter and without the $C$-filter. Performance of closed loop system with and without the colouring filter in the disturbance model was compared.

\section{Model of the controlled system}

Let us consider a two input - two output system. The two - input/two - output (TITO) processes are the most often encountered multivariable processes in practice and many processes with inputs/outputs beyond two can be treated as several TITO subsystems [11].

A general transfer matrix of a two-input-two-output system with significant cross-coupling between the control loops is expressed as: 


$$
\begin{gathered}
\boldsymbol{G}(z)=\left[\begin{array}{ll}
G_{11}(z) & G_{12}(z) \\
G_{21}(z) & G_{22}(z)
\end{array}\right] \\
\boldsymbol{Y}(z)=\boldsymbol{G}(z) \boldsymbol{U}(z)
\end{gathered}
$$

where $\boldsymbol{U}(z)$ and $\boldsymbol{Y}(z)$ are vectors of the manipulated variables and the controlled variables, respectively.

$$
\boldsymbol{U}(z)=\left[u_{1}(z), u_{2}(z)\right]^{T} \boldsymbol{Y}(z)=\left[y_{1}(z), y_{2}(z)\right]^{T}
$$

It may be assumed that the transfer matrix can be transcribed to the following form of the matrix fraction:

$$
\boldsymbol{G}(z)=\boldsymbol{A}^{-1}\left(z^{-1}\right) \boldsymbol{B}\left(z^{-1}\right)=\boldsymbol{B}_{1}\left(z^{-1}\right) \boldsymbol{A}_{1}^{-1}\left(z^{-1}\right)
$$

where the polynomial matrices $\boldsymbol{A} \in R_{22}\left[z^{-1}\right], \boldsymbol{B} \in R_{22}\left[z^{-1}\right]$ are the left coprime factorizations of matrix $\boldsymbol{G}(z)$ and the matrices $A_{1} \in R_{22}\left[z^{-1}\right], B_{1} \in R_{22}\left[z^{-1}\right]$ are the right coprime factorizations of $\boldsymbol{G}(z)$. The model can be also written in the form

$$
\boldsymbol{A}\left(z^{-1}\right) \boldsymbol{Y}(z)=\boldsymbol{B}\left(z^{-1}\right) \boldsymbol{U}(z)
$$

As an example a model with polynomials of second degree was chosen. This model proved to be effective for control of several TITO laboratory processes [12], where controllers based on a model with polynomials of the first degree failed. The model has sixteen parameters. The matrices $\boldsymbol{A}$ and $\boldsymbol{B}$ are defined as follows

$$
\begin{gathered}
A\left(z^{-1}\right)=\left[\begin{array}{cc}
1+a_{1} z^{-1}+a_{2} z^{-2} & a_{3} z^{-1}+a_{4} z^{-2} \\
a_{5} z^{-1}+a_{6} z^{-2} & 1+a_{7} z^{-1}+a_{8} z^{-2}
\end{array}\right] \\
\boldsymbol{B}\left(z^{-1}\right)=\left[\begin{array}{ll}
b_{1} z^{-1}+b_{2} z^{-2} & b_{3} z^{-1}+b_{4} z^{-2} \\
b_{5} z^{-1}+b_{6} z^{-2} & b_{7} z^{-1}+b_{8} z^{-2}
\end{array}\right]
\end{gathered}
$$

A widely used model in general model predictive control is the CARIMA (controller autoregressive integrated moving average) model which we can obtain by adding a disturbance model as

$$
\boldsymbol{A}\left(z^{-1}\right) \boldsymbol{y}(k)=\boldsymbol{B}\left(z^{-1}\right) \boldsymbol{u}(k)+\boldsymbol{C}\left(z^{-1}\right) \boldsymbol{\Delta}^{-1}\left(z^{-1}\right) \boldsymbol{n}(k)
$$

where $\boldsymbol{n}$ is a non-measurable random disturbance that is assumed to have zero mean value and constant covariance and

$$
\Delta\left(z^{-1}\right)=\left[\begin{array}{cc}
1-z^{-1} & 0 \\
0 & 1-z^{-1}
\end{array}\right]
$$

in case of TITO system. $\boldsymbol{C}$ is the colouring polynomial matrix. For purpose of simplification it is often supposed to be equal to the identity matrix [3]. In a single input single output case we have a colouring polynomial $C$ instead of the matrix $\boldsymbol{C}$.

$$
\Delta A\left(z^{-1}\right) y(k)=B\left(z^{-1}\right) \Delta u(k)+C\left(z^{-1}\right) n(k)
$$

where $A, B$ and $C$ are polynomials and $\Delta=1-z^{-1}$. In Model Predictive Control it is also common to treat $C$ as a design parameter [6], [7], [13]. Analogically the polynomial matrix $\boldsymbol{C}$ could be expected as the design parameters in a multivariable case. Nevertheless considering the polynomial matrix $\boldsymbol{C}$ as the design parameter is computationally unsolvable and practically inapplicable. A simplified model when the nonmeasurable random disturbance was a scalar was then considered

$$
\boldsymbol{\Delta} \boldsymbol{A}\left(z^{-1}\right) \boldsymbol{y}(k)=\boldsymbol{B}\left(z^{-1}\right) \boldsymbol{\Delta} \boldsymbol{u}(k)+C\left(z^{-1}\right) n(k)
$$

Further will be compared cases when $\boldsymbol{C}$ is the identity matrix and when the input and output variables are filtered with a colouring polynomial $C$ which is supposed as the design parameter.

\section{Implementation of predictive controller}

The basic idea of MPC is to use a model of a controlled process to predict $N$ future outputs of the process. A trajectory of future manipulated variables is given by solving an optimization problem incorporating a suitable cost function and constraints. Only the first element of the obtained control sequence is applied. The whole procedure is repeated in following sampling period. This principle is known as the receding horizon strategy. The computation of a control law of MPC is based on minimization of the following criterion

$$
J(k)=\sum_{j=N_{1}}^{N} \boldsymbol{e}(k+j)^{2}+\lambda \sum_{j=1}^{N_{u}} \Delta \boldsymbol{u}(k+j)^{2}
$$

where $\boldsymbol{e}(k+j)$ is a vector of predicted control errors, $\Delta \boldsymbol{u}(k+j)$ is a vector of future increments of the manipulated variable (for the system with two inputs and two outputs each vector has two elements), $N$ is a length of the prediction horizon, $N_{u}$ is a length of the control horizon and $\lambda$ is a weighting factor of control increments. A predictor in a vector form is given by

$$
\hat{\boldsymbol{y}}=\boldsymbol{G} \Delta \boldsymbol{u}+\boldsymbol{y}_{0}
$$

where $\hat{\boldsymbol{y}}$ is a vector of system predictions along the horizon of the length $N, \Delta \boldsymbol{u}$ is a vector of control increments, $\boldsymbol{y}_{0}$ is the free response vector. $\boldsymbol{G}$ is a matrix of the dynamics. It is given as

$$
\boldsymbol{G}=\left[\begin{array}{ccccc}
\boldsymbol{G}_{0} & 0 & \cdots & \cdots & 0 \\
\boldsymbol{G}_{1} & \boldsymbol{G}_{0} & 0 & \cdots & 0 \\
\vdots & & \ddots & \ddots & \vdots \\
\vdots & & & \boldsymbol{G}_{0} & 0 \\
\boldsymbol{G}_{N-1} & \cdots & \cdots & \cdots & \boldsymbol{G}_{0}
\end{array}\right]
$$

where sub-matrices $\boldsymbol{G}_{i}$ have dimension $2 \times 2$ and contain values of the step sequence.

The criterion (12) can be written in a general vector form

$$
J=(\hat{y}-w)^{T}(\hat{y}-w)+\lambda \Delta \boldsymbol{u}^{T} \Delta \boldsymbol{u}
$$

where $\boldsymbol{w}$ is a vector of the reference trajectory. The criterion can be modified using the expression (15) to

$$
J=2 \boldsymbol{g}^{T} \Delta \boldsymbol{u}+\Delta \boldsymbol{u}^{T} \boldsymbol{H} \Delta \boldsymbol{u}
$$

where the gradient $\boldsymbol{g}$ and the Hess matrix $\boldsymbol{H}$ are defined by following expressions

$$
\begin{gathered}
\boldsymbol{g}^{T}=\boldsymbol{G}^{T}\left(\boldsymbol{y}_{0}-\boldsymbol{w}\right) \\
\boldsymbol{H}=\boldsymbol{G}^{T} \boldsymbol{G}+\lambda \boldsymbol{I}
\end{gathered}
$$

Handling of constraints is one of main advantages of predictive control. General formulation of predictive control with constraints is then as follows

$$
\min _{\Delta u} 2 \boldsymbol{g}^{T} \Delta \boldsymbol{u}+\Delta \boldsymbol{u}^{T} \boldsymbol{H} \Delta \boldsymbol{u}
$$

owing to 
$\boldsymbol{A} \Delta \boldsymbol{u} \leq \boldsymbol{b}$

The inequality (20) expresses the constraints in a compact form.

\section{Computation of Predictions $C=I$}

An important task in predictive control is computation of predictions for arbitrary prediction and control horizons.

The difference equation of the CARIMA model without the unknown term can be expressed as:

$$
\begin{aligned}
& y_{1}(k+1)=\left(1-a_{1}\right) y_{1}(k)+\left(a_{1}-a_{2}\right) y_{1}(k-1)+a_{2} y_{1}(k-2)- \\
& -a_{3} y_{2}(k)+\left(a_{3}-a_{4}\right) y_{2}(k-1)+a_{4} y_{2}(k-2)+ \\
& +b_{1} \Delta u_{1}(k)+b_{2} \Delta u_{1}(k-1)+b_{3} \Delta u_{2}(k)+b_{4} \Delta u_{2}(k-1) \\
& y_{2}(k+1)=\left(1-a_{7}\right) y_{2}(k)+\left(a_{7}-a_{8}\right) y_{2}(k-1)+a_{8} y_{2}(k-2)- \\
& -a_{5} y_{1}(k)+\left(a_{5}-a_{6}\right) y_{1}(k-1)+a_{6} y_{1}(k-2)+ \\
& +b_{5} \Delta u_{1}(k)+b_{6} \Delta u_{1}(k-1)+b_{7} \Delta u_{2}(k)+b_{8} \Delta u_{2}(k-1)
\end{aligned}
$$

These equations can be written into a matrix form

$$
\begin{aligned}
& \boldsymbol{y}(k+1)=\boldsymbol{A}_{1} \boldsymbol{y}(k)+\boldsymbol{A}_{2} \boldsymbol{y}(k-1)+\boldsymbol{A}_{3} \boldsymbol{y}(k-2)+ \\
& +\boldsymbol{B}_{1} \Delta \boldsymbol{u}(k)+\boldsymbol{B}_{2} \Delta \boldsymbol{u}(k-1)
\end{aligned}
$$

where

$$
\begin{gathered}
\boldsymbol{A}_{1}=\left[\begin{array}{cc}
1-a_{1} & -a_{3} \\
-a_{5} & 1-a_{7}
\end{array}\right] \boldsymbol{A}_{2}=\left[\begin{array}{ll}
a_{1}-a_{2} & a_{3}-a_{4} \\
a_{5}-a_{6} & a_{7}-a_{8}
\end{array}\right] \\
\boldsymbol{A}_{3}=\left[\begin{array}{ll}
a_{2} & a_{4} \\
a_{6} & a_{8}
\end{array}\right] \\
\boldsymbol{B}_{1}=\left[\begin{array}{ll}
b_{1} & b_{3} \\
b_{5} & b_{7}
\end{array}\right] \boldsymbol{B}_{2}=\left[\begin{array}{ll}
b_{2} & b_{4} \\
b_{6} & b_{8}
\end{array}\right]
\end{gathered}
$$

It was necessary to directly compute three steps-ahead predictions in a straightforward way by establishing of previous predictions to later predictions. The model order defines that computation of one step-ahead prediction is based on the three past values of the system output.

$$
\begin{aligned}
& \hat{\boldsymbol{y}}(k+1)=\boldsymbol{A}_{1} \boldsymbol{y}(k)+\boldsymbol{A}_{2} \boldsymbol{y}(k-1)+\boldsymbol{A}_{3} \boldsymbol{y}(k-2)+ \\
& +\boldsymbol{B}_{1} \Delta \boldsymbol{u}(k)+\boldsymbol{B}_{2} \Delta \boldsymbol{u}(k-1) \\
& \hat{\boldsymbol{y}}(k+2)=\boldsymbol{A}_{1} \boldsymbol{y}(k+1)+\boldsymbol{A}_{2} \boldsymbol{y}(k)+\boldsymbol{A}_{3} \boldsymbol{y}(k-1)+ \\
& +\boldsymbol{B}_{1} \Delta \boldsymbol{u}(k+1)+\boldsymbol{B}_{2} \Delta \boldsymbol{u}(k) \\
& \hat{\boldsymbol{y}}(k+3)=\boldsymbol{A}_{1} \boldsymbol{y}(k+2)+\boldsymbol{A}_{2} \boldsymbol{y}(k+1)+\boldsymbol{A}_{3} \boldsymbol{y}(k)+ \\
& +\boldsymbol{B}_{1} \Delta \boldsymbol{u}(k+2)+\boldsymbol{B}_{2} \Delta \boldsymbol{u}(k+1)
\end{aligned}
$$

It is possible to divide computation of the predictions to recursion of the free response and recursion of the matrix of the dynamics. The free response vector can be expressed as:

$$
\begin{aligned}
& \boldsymbol{y}_{0}=\left[\begin{array}{ll}
p_{11} & p_{12} \\
p_{21} & q_{22} \\
\hline p_{31} & p_{32} \\
p_{41} & p_{42} \\
\hline p_{51} & p_{52} \\
p_{61} & p_{62}
\end{array}\right]\left[\begin{array}{ll}
\Delta u_{1}(k-1) \\
\Delta u_{2}(k-1)
\end{array}\right]+\left[\begin{array}{ll|ll|ll}
q_{11} & q_{12} & q_{13} & q_{14} & q_{15} & q_{16} \\
q_{21} & q_{22} & q_{23} & q_{24} & q_{25} & q_{26} \\
\hline q_{31} & q_{32} & q_{33} & q_{34} & q_{35} & q_{36} \\
q_{41} & q_{42} & q_{43} & q_{44} & q_{45} & q_{46} \\
\hline q_{51} & q_{52} & q_{53} & q_{54} & q_{55} & q_{56} \\
q_{61} & q_{62} & q_{63} & q_{64} & q_{65} & q_{66}
\end{array}\right]\left[\begin{array}{c}
y_{1}(k) \\
y_{2}(k) \\
y_{1}(k-1) \\
y_{2}(k-1) \\
y_{1}(k-2) \\
y_{2}(k-2)
\end{array}\right]= \\
& =\left[\begin{array}{l}
\boldsymbol{P}_{1} \\
\boldsymbol{P}_{2} \\
\boldsymbol{P}_{3}
\end{array}\right] \Delta \boldsymbol{u}(k-1)+\left[\begin{array}{lll}
\boldsymbol{Q}_{11} & \boldsymbol{Q}_{12} & \boldsymbol{Q}_{13} \\
\boldsymbol{Q}_{21} & \boldsymbol{Q}_{22} & \boldsymbol{Q}_{23} \\
\boldsymbol{Q}_{31} & \boldsymbol{Q}_{32} & \boldsymbol{Q}_{33}
\end{array}\right]\left[\begin{array}{c}
\boldsymbol{y}(k) \\
\boldsymbol{y}(k-1) \\
\boldsymbol{y}(k-2)
\end{array}\right]=\boldsymbol{P} \Delta \boldsymbol{u}(k-1)+\boldsymbol{Q}\left[\begin{array}{c}
\boldsymbol{y}(k) \\
\boldsymbol{y}(k-1) \\
\boldsymbol{y}(k-2)
\end{array}\right]
\end{aligned}
$$

The coefficients of the matrices $\boldsymbol{P}$ and $\boldsymbol{Q}$ for further predictions are computed recursively. Based on the three previous predictions it is repeatedly computed the next row of the matrices $\boldsymbol{P}$ and $\boldsymbol{Q}$ in the following way:

$$
\begin{gathered}
\boldsymbol{P}_{4}=\left[\begin{array}{ll}
p_{71} & p_{72} \\
p_{81} & p_{82}
\end{array}\right]=\boldsymbol{A}_{1} \boldsymbol{P}_{31}+\boldsymbol{A}_{2} \boldsymbol{P}_{21}+\boldsymbol{A}_{3} \boldsymbol{P}_{11} \\
\boldsymbol{Q}_{41}=\left[\begin{array}{ll}
q_{71} & q_{72} \\
q_{81} & q_{82}
\end{array}\right]=\boldsymbol{A}_{1} \boldsymbol{Q}_{31}+\boldsymbol{A}_{2} \boldsymbol{Q}_{21}+\boldsymbol{A}_{3} \boldsymbol{Q}_{11} \\
\boldsymbol{Q}_{42}=\left[\begin{array}{ll}
q_{73} & q_{74} \\
q_{83} & q_{84}
\end{array}\right]=\boldsymbol{A}_{1} \boldsymbol{Q}_{32}+\boldsymbol{A}_{2} \boldsymbol{Q}_{22}+\boldsymbol{A}_{3} \boldsymbol{Q}_{12} \\
\boldsymbol{Q}_{43}=\left[\begin{array}{ll}
q_{75} & q_{76} \\
q_{85} & q_{86}
\end{array}\right]=\boldsymbol{A}_{1} \boldsymbol{Q}_{33}+\boldsymbol{A}_{2} \boldsymbol{Q}_{23}+\boldsymbol{A}_{3} \boldsymbol{Q}_{13}
\end{gathered}
$$

The recursion of the matrix $\boldsymbol{G}$ is similar. The next element of the first column is repeatedly computed and the remaining columns are shifted. This procedure is performed repeatedly until the prediction horizon is achieved. If the control horizon is lower than the prediction horizon a number of columns in the matrix is reduced. The technique is apparent from the equations (30) and (31).

$$
\begin{gathered}
\boldsymbol{G} \Delta \boldsymbol{u}=\left[\begin{array}{cc|cc}
g(1,1) & g(1,2) & 0 & 0 \\
g(2,1) & g(2,2) & 0 & 0 \\
\hline g(3,1) & g(3,2) & g(1,1) & g(1,2) \\
g(4,1) & g(4,2) & g(2,1) & g(2,2) \\
\hline g(5,1) & g(5,2) & g(3,1) & g(3,2) \\
g(6,1) & g(6,2) & g(4,1) & g(4,2)
\end{array}\right]\left[\begin{array}{c}
\Delta u_{1}(k) \\
\Delta u_{2}(k) \\
\Delta u_{1}(k+1) \\
\Delta u_{2}(k+1)
\end{array}\right]= \\
=\left[\begin{array}{cc}
G(1,1) & 0 \\
G(2,1) & G(1,1) \\
G(3,1) & G(2,1)
\end{array}\right]\left[\begin{array}{c}
\Delta \boldsymbol{u}(k) \\
\Delta \boldsymbol{u}(k+1)
\end{array}\right] \\
\boldsymbol{G}_{41}=\left[\begin{array}{cc}
g_{71} & g_{72} \\
g_{81} & g_{82}
\end{array}\right]=\boldsymbol{A}_{1} \boldsymbol{G}_{31}+\boldsymbol{A}_{2} \boldsymbol{G}_{21}+\boldsymbol{A}_{3} \boldsymbol{G}_{11}
\end{gathered}
$$
form

The predictions can be written in a compact matrix

$$
\begin{aligned}
& \hat{\boldsymbol{y}}(k+j)=\boldsymbol{G} \Delta \boldsymbol{u}(k+j-1)+\boldsymbol{P} \Delta \boldsymbol{u}(k-1)+\boldsymbol{Q} \boldsymbol{y}(k-j+1) \\
& j \leq N
\end{aligned}
$$

\section{Computation of predictions with colouring filter $C$}

Computation of predictions with colouring filter is solved for example in [14] for a single input-single output case. Including the $C$-filter the CARIMA model takes the form

$$
\boldsymbol{\Delta} \boldsymbol{A}\left(z^{-1}\right) \boldsymbol{y}(k)=\boldsymbol{B}\left(z^{-1}\right) \boldsymbol{\Delta} \boldsymbol{u}(k)+C\left(z^{-1}\right) n(k)
$$

As it was previously mentioned, a simplified model when the non-measurable random disturbance was a scalar was considered.

Equation (33) can be modified to

$$
\boldsymbol{\Delta} \boldsymbol{A}\left(z^{-1}\right) \boldsymbol{y}(k) \frac{1}{C\left(z^{-1}\right)}=\boldsymbol{B}\left(z^{-1}\right) \boldsymbol{\Delta} \boldsymbol{u}(k) \frac{1}{C\left(z^{-1}\right)}+n(k)
$$

where the unknown term is supposed to be the white noise and the input and output variables are filtered. Using of (34) for prediction improves prediction accuracy.

The filtered variables are defined as 


$$
\begin{aligned}
& \left(\begin{array}{l}
y_{1 f}(k) \\
y_{2 f}(k)
\end{array}\right)=\left(\begin{array}{l}
y_{1}(k) \\
y_{2}(k)
\end{array}\right) \frac{1}{C\left(z^{-1}\right)} \\
& \left(\begin{array}{l}
u_{1 f}(k) \\
u_{2 f}(k)
\end{array}\right)=\left(\begin{array}{l}
u_{1}(k) \\
u_{2}(k)
\end{array}\right) \frac{1}{C\left(z^{-1}\right)}
\end{aligned}
$$

In this case the polynomial $C$ is a design parameter. It is a stable polynomial. For the system with polynomials of the second degree (6), (7) it was chosen to be of the second degree as well

$$
C\left(z^{-1}\right)=1+c_{1} z^{-1}+c_{2} z^{-2}
$$

The input and output data are filtered before prediction. $1 / C$ is a low-pass filter which reduces high frequency noise. It is easy to prove by simulation that the cases when the noise is coloured (33) and when the noise is white and the input and output variables are filtered (34) are equal.

The prediction equation for filtered variables takes the following form

$$
\begin{aligned}
& \hat{\boldsymbol{y}}_{f}(k+j)=\boldsymbol{G} \Delta \boldsymbol{u}_{f}(k+j-1)+\boldsymbol{P} \Delta \boldsymbol{u}_{f}(k-1)+\boldsymbol{Q} \boldsymbol{y}_{f}(k-j+1) \\
& j \leq N
\end{aligned}
$$

For practical application equation (28) is inapplicable. Prediction of the unfiltered output must be expressed by means of future control increments.

The relationship between filtered and unfiltered variables can be expressed as follows

$$
\begin{gathered}
\left(\begin{array}{l}
y_{1 f}(k) \\
y_{2 f}(k)
\end{array}\right)=\left(\begin{array}{l}
y_{1}(k) \\
y_{2}(k)
\end{array}\right) \frac{1}{1+c_{1} z^{-1}+c_{2} z^{-2}} \\
\left(\begin{array}{l}
y_{1}(k) \\
y_{2}(k)
\end{array}\right)=\left(\begin{array}{l}
y_{1 f}(k)+c_{1} y_{1 f}(k-1)+c_{2} y_{1 f}(k-2) \\
y_{2 f}(k)+c_{1} y_{2 f}(k-1)+c_{2} y_{2 f}(k-2)
\end{array}\right)
\end{gathered}
$$

For three step ahead predictions

$$
\begin{aligned}
& \hat{\boldsymbol{y}}(k+1)=\boldsymbol{y}_{f}(k+1)+c_{1} \boldsymbol{y}_{f}(k)+c_{2} \boldsymbol{y}_{f}(k-1) \\
& \hat{\boldsymbol{y}}(k+2)=\boldsymbol{y}_{f}(k+2)+c_{1} \boldsymbol{y}_{f}(k+1)+c_{2} \boldsymbol{y}_{f}(k) \\
& \hat{\boldsymbol{y}}(k+3)=\boldsymbol{y}_{f}(k+3)+c_{1} \boldsymbol{y}_{f}(k+2)+c_{2} \boldsymbol{y}_{f}(k+1)
\end{aligned}
$$

In a matrix form the equations (31) can be expressed as follows

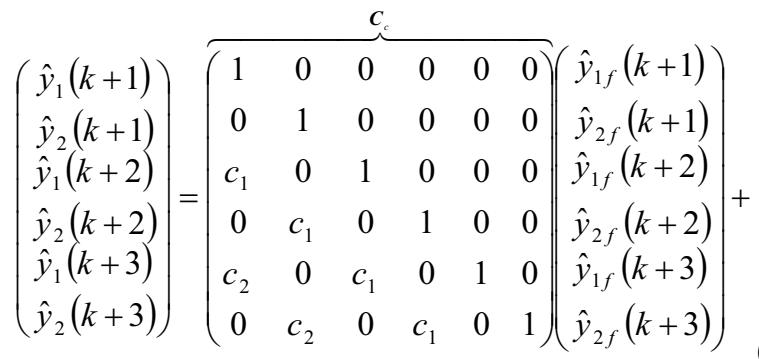

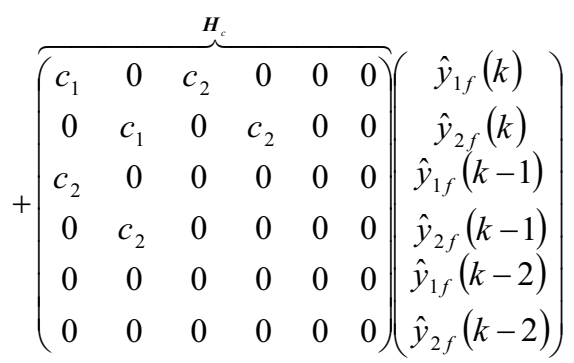

The relationship between filtered and unfiltered control increments can be expressed similarly. Using matrix notation we can define following equations

$$
\begin{array}{r}
\hat{\boldsymbol{y}}(k+j)=\boldsymbol{C}_{C} \boldsymbol{y}_{f}(k+j)+\boldsymbol{H}_{C} \boldsymbol{y}_{f}(k-j+1) \\
\Delta \boldsymbol{u}(k+j)=\boldsymbol{C}_{C} \Delta \boldsymbol{u}_{f}(k+j)+\boldsymbol{H}_{C} \Delta \boldsymbol{u}_{f}(k-j+1)
\end{array}
$$

where matrices $\boldsymbol{C}_{\mathrm{c}}$ and $\boldsymbol{H}_{\mathrm{c}}$ are defined as follows

$$
\begin{aligned}
\boldsymbol{C}_{C} & =\left(\begin{array}{cccccc}
1 & 0 & 0 & 0 & 0 & 0 \\
0 & 1 & 0 & 0 & 0 & 0 \\
c_{1} & 0 & 1 & 0 & 0 & 0 \\
0 & c_{1} & 0 & 1 & 0 & 0 \\
c_{2} & 0 & c_{1} & 0 & 1 & 0 \\
0 & c_{2} & 0 & c_{1} & 0 & 1
\end{array}\right) \\
\boldsymbol{H}_{C} & =\left(\begin{array}{ccccccc}
c_{1} & 0 & c_{2} & 0 & 0 & 0 \\
0 & c_{1} & 0 & c_{2} & 0 & 0 \\
c_{2} & 0 & 0 & 0 & 0 & 0 \\
0 & c_{2} & 0 & 0 & 0 & 0 \\
0 & 0 & 0 & 0 & 0 & 0 \\
0 & 0 & 0 & 0 & 0 & 0
\end{array}\right)
\end{aligned}
$$

From equations (43) and (44) we can express the filtered variables

$$
\begin{aligned}
& \hat{\boldsymbol{y}}(k+j)=\boldsymbol{C}_{C} \boldsymbol{y}_{f}(k+j)+\boldsymbol{H}_{C} \boldsymbol{y}_{f}(k-j+1) \\
& \Delta \boldsymbol{u}(k+j)=\boldsymbol{C}_{C} \Delta \boldsymbol{u}_{f}(k+j)+\boldsymbol{H}_{C} \Delta \boldsymbol{u}_{f}(k-j+1)(48)
\end{aligned}
$$

After substitution of equations (47) and (48) to equation (38) we obtain

$$
\begin{aligned}
& \boldsymbol{C}_{C}{ }^{-1}\left(\hat{\boldsymbol{y}}(k+j)-\boldsymbol{H}_{C} \boldsymbol{y}_{f}(k-j+1)\right)= \\
& =\boldsymbol{G} \boldsymbol{C}_{C}{ }^{-1}\left(\Delta \boldsymbol{u}(k+j-1)-\boldsymbol{H}_{C} \Delta \boldsymbol{u}_{f}(k-1)\right)+ \\
& +\boldsymbol{P} \Delta \boldsymbol{u}_{f}(k-1)+\boldsymbol{Q} \boldsymbol{y}_{f}(k-j+1)
\end{aligned}
$$

After modification we obtain resulting equation of the predictor

$$
\begin{aligned}
& \hat{\boldsymbol{y}}(k+j)=\boldsymbol{G} \Delta \boldsymbol{u}(k+j-1)+\left[\boldsymbol{C}_{C} \boldsymbol{P}-\boldsymbol{G} \boldsymbol{H}_{C}\right] \Delta \boldsymbol{u}_{f}(k-1)+ \\
& +\left[\boldsymbol{H}_{C}+\boldsymbol{C}_{C} \boldsymbol{Q}\right] \boldsymbol{y}_{f}(k-j+1)
\end{aligned}
$$

We can establish following substitutions

$$
\begin{array}{r}
\widetilde{\boldsymbol{P}}=\left[\boldsymbol{C}_{C} \boldsymbol{P}-\boldsymbol{G} \boldsymbol{H}_{C}\right] \\
\widetilde{\boldsymbol{Q}}=\left[\boldsymbol{H}_{C}+\boldsymbol{C}_{C} \boldsymbol{Q}\right]
\end{array}
$$
form

The prediction equation then can be written in the

$$
\hat{\boldsymbol{y}}(k+j)=\boldsymbol{G} \Delta \boldsymbol{u}(k+j-1)+\widetilde{\boldsymbol{P}} \Delta \boldsymbol{u}_{f}(k-1)+\widetilde{\boldsymbol{Q}} \boldsymbol{y}_{f}(k-j+1)(53
$$

\section{Simulation verification}

Verification by simulation was carried out on a range of plants with various dynamics. The control of the model below is given here as an example.

$$
\boldsymbol{G}(s)=\left[\begin{array}{cc}
\frac{3}{5 s^{2}+6 s+1} & \frac{2}{2 s^{2}+4 s+1} \\
\frac{7}{3 s^{2}+10 s+1} & \frac{5}{2 s^{2}+8 s+1}
\end{array}\right]
$$

A corresponding discrete model in the form given by equations (6), (7) and (21) was obtained by recursive identification. Control in the initial adaptation phase then has worse quality. It does not exist a systematic way for 
selection of the filter $C$. Its selection is mostly based on intuition. In our example the filter was chosen as

$$
C\left(z^{-1}\right)=1+0,8 z^{-1}+0,05 z^{-2}
$$

The sampling period was tuned experimentally and the best value was $T_{0}=0,5 \mathrm{~s}$. The controlled variable was affected by a noise with zero mean value and constant covariance. Simulation sampling of noise was $0,1 \mathrm{~s}$.

In figures 1-6 are simulation results. Figures 1,3 and 5 show time responses of the control without the filtering of the variables introduced in section 4. Figures 2, 4 and 6 show time responses of the control with the filtering of variables described in section 5 .

In figures 1 and 2 there is the response of the controlled variable taken by $0,1 s$. It means with the same sampling period as the simulation noise. Simulation results in this figure are the closest to the reality. In figures 3 and 4 there is the controlled variable taken by 2 $s$. It means with the same sampling period which is used for the control. The data then simulates measured values. In figures 5 and 6 is the manipulated variable.

The tuning parameters that are lengths of the prediction and control horizons and the weighting coefficient $\lambda$ were tuned experimentally. There is a lack of clear theory relating to the closed loop behavior to design parameters. The length of the prediction horizon, which should cover the important part of the step response, was set to $N=5$. The length of the control horizon was also set to $N_{u}=5$. The coefficient $\lambda$ was taken as equal to 0,1 .

It is necessary to emphasize that the displayed inputs and outputs in the graphs are not filtered. The filtered values are used only for computation of systems output predictions and consequently for computation of the control law. The displayed inputs and outputs are real unfiltered values.
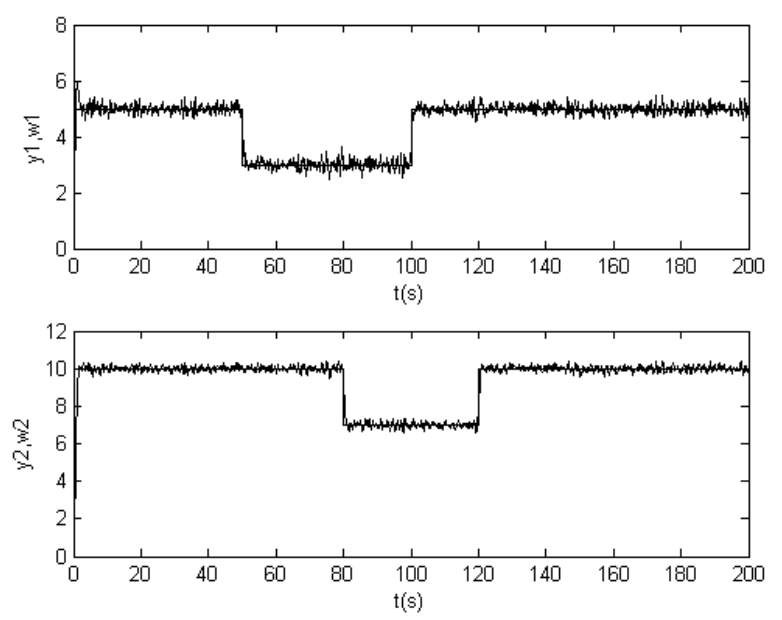

Figure 1. Controlled variables sampled by $0,1 s$ - case without filtering of variables
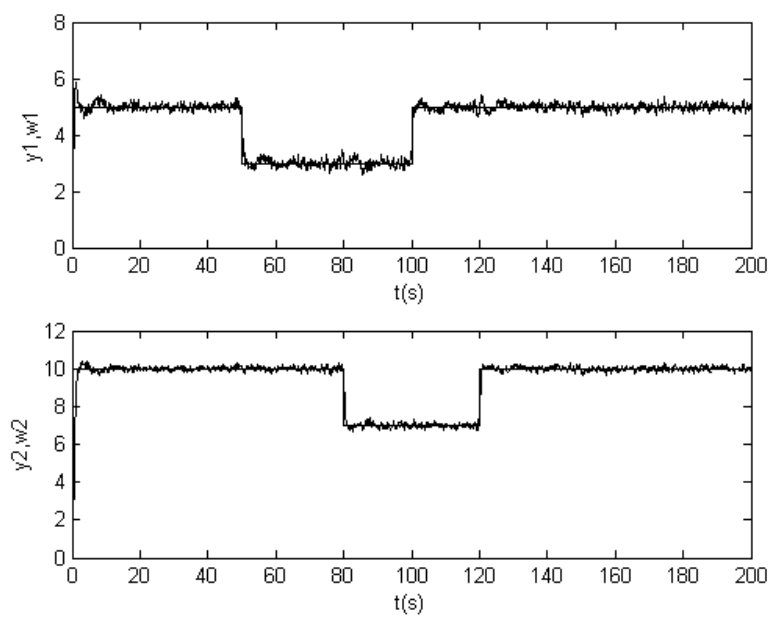

Figure 2. Controlled variables sampled by $0,1 s$ - case with filtering of variables
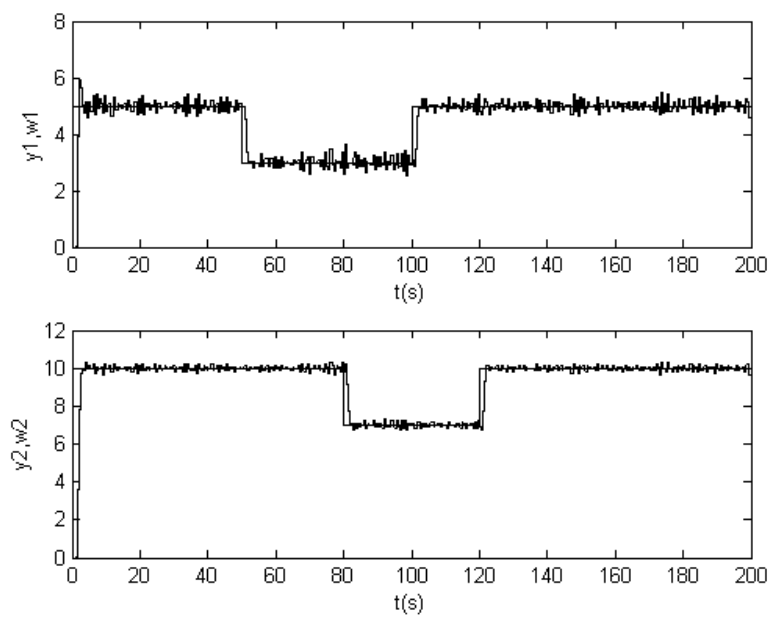

Figure 3. Controlled variables sampled by $0,5 s-$ case without filtering of variables
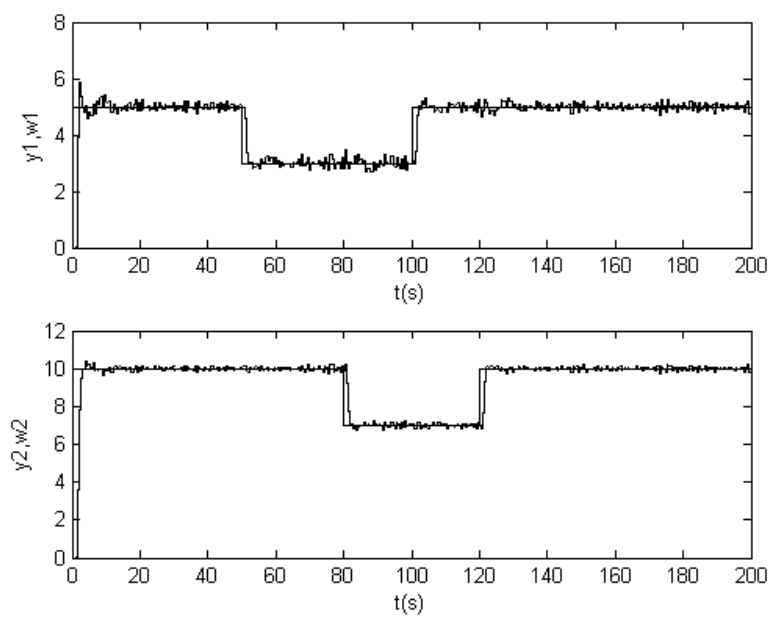

Figure 4. Controlled variables sampled by $0,5 s-$ case with filtering of variables 

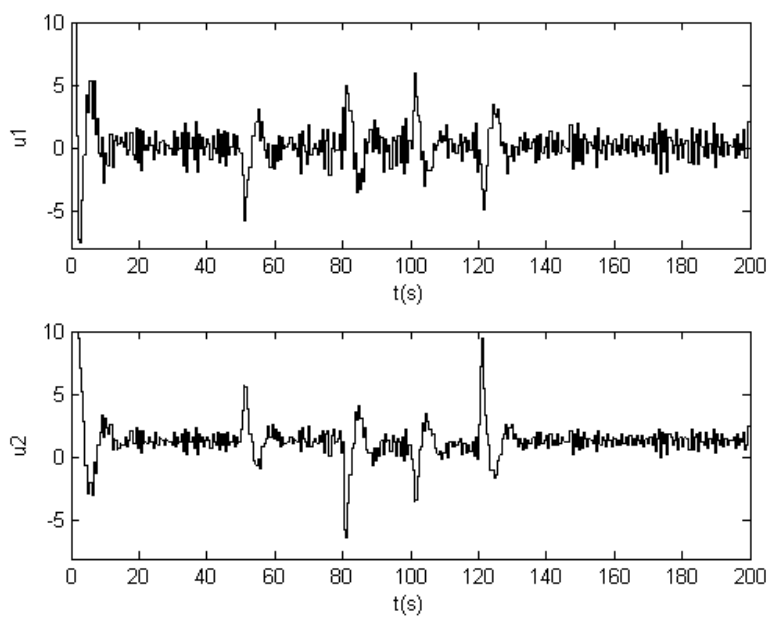

Figure 5. Manipulated variables - case without filtering of variables
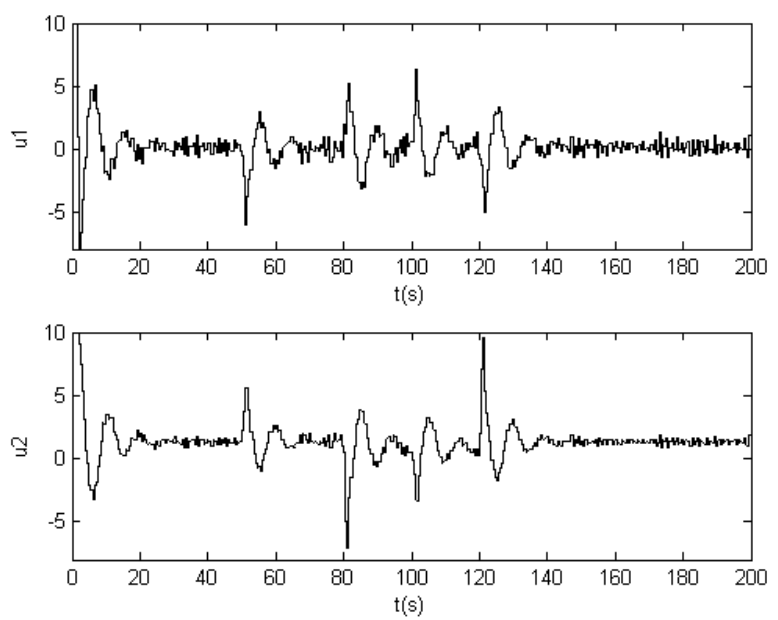

Figure 6. Manipulated variables - case with filtering of variables

\section{Conclusion}

Specific self-contained prediction equations for the inputoutput model in the form of matrix fraction were derived for the case with filtering of the input and output variables. Simulations, where the filtered variables are used for computation of the control law and the manipulated variable, were performed. In the simulation results are displayed real unfiltered variables. By simulation control of a range of systems were compared control results of cases with and without the C-filter. In the paper there is introduced one simulation example. The best achieved results are shown. The C-filter is a tuning parameter for which setting we do not have available any exact methodology. The filter was designed by try it and see approach as a low pass filter. Obviously better results were achieved in case with the C-filter particularly regarding rate of oscillations of the input and output variables. It is obvious that the variables are more settled in case with the $\mathrm{C}$-filter. The filter reduces sensitivity of the closed loop system to high frequency noise. Cost for this improvement is a relatively difficult setting of the $\mathrm{C}$-filter as a parameter.

\section{Acknowledgement}

This work was supported by the Ministry of Education, Youth and Sports of the Czech Republic within the National Sustainability Programme project No. LO1303 (MSMT-7778/2014) and also by the European Regional Development Fund under the project CEBIA-Tech No. CZ.1.05/2.1.00/03.0089 and also by the Programme EEA and Norway Grants for funding via grant on Institutional cooperation project nr. NF-CZ07-ICP-4-345-2016

\section{References}

1. P.R. Krishnawamy, et al., Ind. Eng. Chem. Res., 30, 662 (1991)

2. W. L. Luyben, Ing. Eng. Chem. Process Des. Dev., 25, 654 (1986)

3. E.F. Camacho, C. Bordons, Model Predictive Control (Springer-Verlag, London, 2004)

4. M. Morari, J.H. Lee, Computers and Chemical Engineering, 23, 667 (1999)

5. J. Mikleš, M. Fikar, Process Modelling, Optimisation and Control (Berlin: Springer-Verlag, 2008)

6. D. W. Clarke, C. Mohtadi, P. S. Tuffs, Automatica, 23,137 (1987)

7. D. W. Clarke, C. Mohtadi, P. S. Tuffs, Automatica, 23,149 (1987)

8. S.J. Quin, T.A. Bandgwell, Proceedings of the Chemical Process Control - V., 93, 232 (1996)

9. S.J. Quin, T.A. Bandgwell, Nonlinear Model Predictive Control (F. Allgöwer \& A. Zheng, Ed.), 369 (2000)

10. S.J. Quin, T.A. Bandgwell, Control Engineering Practice, 11, 733 (2003)

11. F. G. Shinskey, Process Control System (McGrawHill. New York, 1996)

12. M. Kubalčík, V. Bobál, IMechE Part I: J. Systems and Control Engineering, 220(I7), 641 (2006)

13. T.W. Yoon, D.W. Clarke, International Journal of Control, 61, 171 (1995)

14. J.A. Rossiter, Model Based Predictive Control: a Practical Approach (CRC Press, 2003) 